\title{
Nemytskii Operator in the Space of Set-Valued Functions of Bounded $\varphi$-Variation
}

\author{
Wadie Aziz \\ Departamento de Fsica y Matemática, Universidad de Los Andes, Trujillo, RBV \\ Email: wadie@ula.ve
}

Received June 13, 2013; revised July 23, 2013, accepted August 19, 2013

Copyright (C) 2013 Wadie Aziz. This is an open access article distributed under the Creative Commons Attribution License, which permits unrestricted use, distribution, and reproduction in any medium, provided the original work is properly cited.

\begin{abstract}
In this paper we consider the Nemytskii operator, i.e., the composition operator defined by $(N f)(t)=H(t, f(t))$, where $H$ is a given set-valued function. It is shown that if the operator $N$ maps the space of functions bounded $\varphi_{1}$-variation in the sense of Riesz with respect to the weight function $\alpha$ into the space of set-valued functions of bounded $\varphi_{2}$-variation in the sense of Riesz with respect to the weight, if it is globally Lipschitzian, then it has to be of the form $(N f)(t)=A(t) f(t)+B(t)$, where $A(t)$ is a linear continuous set-valued function and $B$ is a set-valued function of bounded $\varphi_{2}$-variation in the sense of Riesz with respect to the weight.
\end{abstract}

Keywords: Bounded Variation; Function of Bounded Variation in the Sense of Riesz; Variation Space; Weight Function; Banach Space; Algebra Space

\section{Introduction}

In [1], it was proved that every globally Lipschitz $\mathrm{Ne}-$ mytskii operator

$$
(N u)(t)=H(t, u(t))
$$

mapping the space $\operatorname{Lip}([a, b] ; c c(Y))$ into itself admits the following representation:

$$
\begin{aligned}
& (N u)(t)=A(t) u(t)+B(t), \\
& u \in \operatorname{Lip}([a, b] ; c c(Y)), t \in[a, b],
\end{aligned}
$$

where $A(t)$ is a linear continuous set-valued function and $B$ is a set-valued function belonging to the space $\operatorname{Lip}([a, b] ; c c(Y))$. The first such theorem for singlevalued functions was proved in [2] on the space of Lipschitz functions. A similar characterization of the Nemytskii operator has also been obtained in [3] on the space of set-valued functions of bounded variation in the classical Jordan sense. For single-valued functions it was proved in [4]. In [5,6], an analogous theorem in the space of set-valued functions of bounded $p$-variation in the sense of Riesz was obtained. Also, they proved a similar result in the case in which that the Nemytskii operator $N$ maps the space of functions of bounded $p$-variation in the sense of Riesz into the space of set-valued functions of bounded $q$-variation in the sense of Riesz, where $1 \leq q \leq p<\infty$, and $N$ is globally Lipschitz. In [7], they showed a similar result in the case where the Nemytskii operator $N$ maps the space $R V_{\varphi_{1}}([a, b] ; K)$ of setvalued functions of bounded $\varphi_{1}$-variation in the sense of Riesz into the space $R W_{\varphi_{2}}([a, b] ; c c(Y))$ of set-valued functions of bounded $\varphi_{2}$-variation in the sense of Riesz and $N$ is globally Lipschitz.

While in [8], we generalize article [6] by introducing a weight function. Now, we intend to generalize [7] in a similar form we did in [8], i.e., the propose of this paper is proving an analogous result in which the Nemytskii operator $N$ maps the space $R V_{\varphi_{1}, \alpha}([a, b] ; K)$ of setvalued functions of bounded $\varphi_{1}$-variation in the sense of Riesz with a weight $\alpha$ into the space

$R W_{\varphi_{2}, \alpha}([a, b] ; c c(Y))$ of set-valued functions of bounded $\varphi_{2}$-variation in the sense of Riesz with a weight $\alpha$ and $N$ is globally Lipschitz.

\section{Preliminary Results}

In this section, we introduce some definitions and recall known results concerning the Riesz $\varphi$-variation.

Definition 2.1 By a $\varphi$-function we mean any nondecreasing continuous function $\varphi:[0,+\infty) \rightarrow[0,+\infty)$ 
such that $\varphi(x)=0$ if and only if $x=0$, and $\varphi(x) \rightarrow \infty$ as $x \rightarrow \infty$.

Let $\mathcal{N}$ be the set of all convex continuous functions that satisfy Definition 2.1.

Definition 2.2 Let $(X,\|\cdot\|)$ be a normed space and $\varphi$ be a $\varphi$-function. Given $I \subset \mathbb{R}$ be an arbitrary (i.e., closed, half-closed, open, bounded or unbounded) fixed interval and $\alpha: I \rightarrow \mathbb{R}$ a fixed continuous strictly increasing function called $a$ it is weight. If $\varphi \in \mathcal{N}$, we define the (total) generalized $\varphi$-variation $V_{\varphi}(f) \equiv$ $V_{\varphi}(f, I, \alpha)$ of the function $f: I \rightarrow X$ with respect to the weight function $\alpha$ in two steps as follows (cf. [9]). If $I=[a, b]$ is a closed interval and $\pi$ is a partition $\pi: a=t_{0}<t_{1}<\cdots<t_{n}=b$ of the interval I (i.e., $\left.n \in \mathbb{N}\right)$, we set

$$
V_{\varphi}(f, \pi, \alpha):=\sum_{i=1}^{n} \varphi\left(\frac{\left\|f\left(t_{i}\right)-f\left(t_{i-1}\right)\right\|}{\left|\alpha\left(t_{i}\right)-\alpha\left(t_{i-1}\right)\right|}\right)\left|\alpha\left(t_{i}\right)-\alpha\left(t_{i-1}\right)\right| .
$$

Denote by $\Pi$ the set of all partitions of $[a, b]$, we set

$$
V_{\varphi}(f) \equiv V_{\varphi}(f,[a, b], \alpha):=\sup \left\{V_{\phi}(f, \pi, \alpha): \pi \in \Pi\right\} .
$$

If $I$ is any interval in $\mathbb{R}$, we put

$$
V_{\varphi}(f) \equiv V_{\varphi}(f, I, \alpha):=\sup \left\{V_{\varphi}(f,[a, b], \alpha):[a, b] \in I\right\} .
$$

The set of all functions of bounded generalized $\varphi$ variation with weight $\alpha$ will be denoted by

$$
\begin{gathered}
R V_{\varphi}(I) \equiv R V_{\varphi}(I, \alpha)=\left\{f:[a, b] \rightarrow X \mid V_{\varphi}(f, I, \alpha)<\infty\right\} . \\
\quad \text { If } \alpha(t)=i \mathrm{~d}(t)=t, t \in I=[a, b], \text { and } \varphi(\rho)=\rho^{q},
\end{gathered}
$$
$\rho \geq 0, q>1$, the $\varphi$-variation $V_{\varphi}(f, I, \alpha)$, also written as $V_{q}(f)$, is the classical $q$-variation of $f$ in the sense of Riesz [10], showing that $V_{q}(f)<\infty$ if and only if $f \in A C(I)$ (i.e., $f: I \rightarrow \mathbb{R}$ is absolutely continuous) and its almost everywhere derivative $f^{\prime}$ is Lebesgue $q$-summable on $I$. Recall that, as it is well known, the space $R V_{\varphi}(I)$ with $I, \varphi$ and $\alpha$ as above and endowed with the norm $|f|_{q}=|f(a)|+\left(V_{q}(f)\right)^{1 / q}$ is a Banach algebra for all $q \geq 1$.

Riesz's criterion was extended by Medvedev [11]: if $\varphi \in \mathcal{N}$, then $f \in R V_{\varphi}(I)$ if and only if $f \in A C(I)$ and $\int_{I} \varphi\left(\left|f^{\prime}(t)\right|\right) \mathrm{d} t<\infty$. Functions of bounded generalized $\varphi$-variation with $\varphi \in \mathcal{N}$ and $\alpha=i d$ (also called functions of bounded Riesz-Orlicz $\varphi$-variation) were studied by Cybertowicz and Matuszewska [12]. They showed that if $f \in R V_{\varphi}(I)$, then

$$
V_{\varphi}(f)=\int_{I} \varphi\left(\left|f^{\prime}(t)\right|\right) \mathrm{d} t,
$$

and that the space

$$
G V_{\varphi}(I)=\left\{f \in \mathbb{R}^{I} \text { such that } \lim _{\lambda \rightarrow+0} V_{\varphi}(\lambda f)=0\right\}
$$

is a semi-normed linear space with the LuxemburgNakano (cf. $[13,14])$ seminorm given by

$$
p_{\varphi}(f)=\inf \left\{r>0 \mid V_{\varphi}(f / r) \leq 1\right\} .
$$

Later, Maligranda and Orlicz [15] proved that the space $G V_{\varphi}(I)$ equipped with the norm

$$
\|f\|_{\varphi}=\sup _{t \in I}|f(t)|+p_{\varphi}(f)
$$

is a Banach algebra.

\section{Generalization of Medvedev Lemma}

We need the following definition:

Definition 3.1 Let $\varphi$ be a $\varphi$-function. We say $\varphi$ satisfies condition $\infty_{1}$ if

$$
\limsup \operatorname{su}_{t \rightarrow \infty} \frac{\varphi(t)}{t}=\infty
$$

For $\varphi$ convex, (1) is just $\lim _{t \rightarrow \infty} \varphi(t) / t=\infty$. Clearly, for $i d=1$ the space $R V_{\alpha}(f,[a, b], i d)$ coincides with the classical space $B V(f,[a, b])$ of functions of bounded variation. In the particular case when $X=\mathbb{R}$ and $1<$ $p<\infty$, we have the space $R V_{p, \alpha}(f,[a, b] ; X)$ of functions of bounded Riesz $p$-variation. Let $\left([a, b], \sum, \mu_{\alpha}\right)$ be a measure space with the Lebesgue-Stieltjes measure defined in $\sigma$-algebra $\sum$ and

$$
\begin{aligned}
& L_{p, \alpha}[a, b]:= \\
& \left\{f:[a, b] \rightarrow \mathbb{R} / f \text { is } \mu_{\alpha} \text { integrable and } \int_{a}^{b}|f|^{p} \mathrm{~d} \alpha<+\infty\right\} .
\end{aligned}
$$

Moreover, let $\alpha$ be a function strictly increasing and continuous in $[a, b]$. We say that $E \subset[a, b]$ has $\mu_{\alpha}$ measure 0 , if given $\varepsilon>0$ there is a countable cover $\left\{\left(a_{n}, b_{n}\right) / n \in \mathbb{N}\right\}$ by open intervals of $E$, such that

$$
\sum_{n=1}^{\infty}\left[\alpha\left(b_{n}\right)-\alpha\left(a_{n}\right)\right]<\varepsilon .
$$

Since $\alpha$ is strictly increasing, the concept of " $\mu_{\alpha}$ measure 0 " coincides with the concept of "measure 0 " of Lebesgue. [cf. [16], § 25].

Definition 3.2 (Jef) A function $f:[a, b] \rightarrow \mathbb{R}$ is said to be absolutely continuous with respect to $\alpha$, if for every $\varepsilon>0$, there exists $\delta>0$ such that

$$
\sum_{j=1}^{n} \varphi\left(\left|f\left(b_{j}\right)-f\left(a_{j}\right)\right|\right) \leq \varepsilon,
$$

for every finite number of nonoverlapping intervals $\left(a_{j}, b_{j}\right), j=1, \cdots, n$ with $\left[a_{j}, b_{j}\right] \subset[a, b]$ and

$$
\sum_{j=1}^{n}\left|\alpha\left(b_{j}\right)-\alpha\left(a_{j}\right)\right| \leq \delta .
$$

The space of all absolutely continuous functions $f:[a, b] \rightarrow \mathbb{R}$, with respect to a function $\alpha$ strictly increasing, is denoted by $\alpha-A C$. Also the following 
characterization of $[17,18]$ is well-known:

Lemma 3.3 Let $f \in \alpha-A C[a, b]$. Then $f_{\alpha}^{\prime}$ exists and is finite in $[a, b]$, except on a set of $\mu_{\alpha}$-measure 0 .

Lemma 3.4 Let $f \in \alpha-A C[a, b]$. Then $f_{\alpha}^{\prime}$ is integrable in the sense Lebesgue-Stieltjes and

$$
f(x)=f(a)+(L-S) \int_{a}^{x} f_{\alpha}^{\prime}(t) \mathrm{d} \alpha(t), \quad x \in[a, b] .
$$

Lemma 3.5 Let $\varphi \in \mathcal{N}$ such that satisfies the $\infty_{1}$ condition. If $f \in R V_{\varphi}(f,[a, b], \alpha)$, then $f$ is $\alpha-a b$ solutely continuous in $[a, b]$, i.e.,

$$
R V_{\varphi}(f,[a, b], \alpha) \subset \alpha-A C[a, b] .
$$

Also the following is a generalization of Medvedev Lemma [11]:

Theorem 3.6 (Generalization a Medvedev Lemma) Let $\varphi \in \mathcal{N}$ such that satisfies the $\infty_{1}$ condition, $f:[a, b] \rightarrow X$. Then

1) If $f$ is $\alpha$-absolutely continuous on $[a, b]$ and

$$
\int_{a}^{b} \varphi\left(\left|f_{\alpha}^{\prime}(x)\right|\right) \mathrm{d} \alpha(x)<+\infty,
$$

then

$$
f \in R V_{\varphi}(f,[a, b], \alpha)
$$

and

$$
R V_{\varphi}(f,[a, b], \alpha) \leq \int_{a}^{b} \varphi\left(\left|f_{\alpha}^{\prime}(x)\right|\right) \mathrm{d} \alpha(x) .
$$

2) If $f \in R V_{\varphi}(f,[a, b], \alpha)$ (i.e., $\left.R V_{\varphi}(f)<+\infty\right)$, then $f$ is $\alpha$-absolutely continuous on $[a, b]$ and

$$
\int_{a}^{b} \varphi\left(\left|f_{\alpha}^{\prime}(x)\right|\right) \mathrm{d} \alpha(x) \leq R V_{\varphi}(f,[a, b], \alpha) .
$$

Proof. 1 ) Since $f$ is $\alpha$ absolutely continuous, there exists $f_{\alpha^{\prime}}$ a.e. in $[a, b]$ by Lemma 3.3. Let $t_{1}, t_{2} \in[a, b], t_{1}<t_{2}$

$$
\begin{aligned}
& \varphi\left(\frac{\left|f\left(t_{2}\right)-f\left(t_{1}\right)\right|}{\left|\alpha\left(t_{2}\right)-\alpha\left(t_{1}\right)\right|}|| \alpha\left(t_{2}\right)-\alpha\left(t_{1}\right) \mid\right. \\
& =\varphi\left(\frac{\left|\int_{t_{1}}^{t_{2}} f_{\alpha^{\prime}}(t) \mathrm{d} \alpha(t)\right|}{\left|\alpha\left(t_{2}\right)-\alpha\left(t_{1}\right)\right|}|| \alpha\left(t_{2}\right)-\alpha\left(t_{1}\right) \mid\right.
\end{aligned}
$$

by Lemma 3.4 and $\varphi$ is strictly increasing

$$
\begin{aligned}
& \leq \varphi\left(\frac{\int_{t_{1}}^{t_{2}}\left|f_{\alpha^{\prime}}(t)\right| \mathrm{d} \alpha(t)}{\left|\alpha\left(t_{2}\right)-\alpha\left(t_{1}\right)\right|}\right)\left|\alpha\left(t_{2}\right)-\alpha\left(t_{1}\right)\right| \\
& =\varphi\left(\frac{\int_{t_{1}}^{t_{2}}\left|f_{\alpha^{\prime}}(t)\right| \mathrm{d} \alpha(t)}{\int_{t_{1}}^{t_{2}}|\mathrm{~d} \alpha(t)|}\right)\left|\alpha\left(t_{2}\right)-\alpha\left(t_{1}\right)\right|
\end{aligned}
$$

using the generalized Jenssen's inequality

$$
\begin{aligned}
& \leq \frac{\int_{t_{1}}^{t_{2}} \varphi\left(\left|f_{\alpha^{\prime}}(t)\right|\right) \mathrm{d} \alpha(t)}{\int_{t_{1}}^{t_{2}} \mathrm{~d} \alpha(t)}\left|\alpha\left(t_{2}\right)-\alpha\left(t_{1}\right)\right| \\
& =\int_{t_{1}}^{t_{2}} \varphi\left(\left|f_{\alpha^{\prime}}(t)\right|\right) \mathrm{d} \alpha(t) .
\end{aligned}
$$

Let $\pi: a=t_{0}<\cdots<t_{n}=b$ be any partition of interval $[a, b]$; then

$$
\begin{aligned}
& \sum_{i=1}^{n} \varphi\left(\frac{\left|f\left(t_{2}\right)-f\left(t_{1}\right)\right|}{\left|\alpha\left(t_{2}\right)-\alpha\left(t_{1}\right)\right|}\right)\left|\alpha\left(t_{2}\right)-\alpha\left(t_{1}\right)\right| \\
& \leq \sum_{i=1}^{n} \int_{t_{i-1}}^{t_{j}} \varphi\left(\left|f_{\alpha^{\prime}}(t)\right|\right) \mathrm{d} \alpha(t)=\int_{a}^{b} \varphi\left(\left|f_{\alpha^{\prime}}(t)\right|\right) \mathrm{d} \alpha(t)<\infty,
\end{aligned}
$$

and we have

$$
V_{\varphi}(f,[a, b], \alpha) \leq \int_{a}^{b} \varphi\left(\left|f_{\alpha}^{\prime}(x)\right|\right) \mathrm{d} \alpha(x) .
$$

Thus $f \in R V_{\varphi}(f,[a, b], \alpha)$.

2 ) Let $f \in R V_{\varphi}[a, b]$. Then $f$ is $\alpha$-absolutely continuous on $[a, b]$ by Lemma 3.5 and $f_{\alpha}^{\prime}$ exist a.e. on $[a, b]$.

For every $n \in \mathbb{N}$, we consider

$$
\pi_{n}: a=t_{0, n}<t_{1, n}<\cdots<t_{n, n}=b
$$

a partition of the interval $[a, b]$ define by

$$
t_{i, n}=a+i \frac{b-a}{n}, i=0,1, \cdots, n .
$$

Let $\left\{f_{n}\right\}_{n}$ be a sequence of step functions, defined by $f_{n}:[a, b] \stackrel{n}{\rightarrow} \mathbb{R}$

$$
t \mapsto f_{n}(t)= \begin{cases}\frac{f\left(t_{i+1, n}\right)-f\left(t_{i, n}\right)}{\alpha\left(t_{i+1, n}\right)-\alpha\left(t_{i, n}\right)}, & t_{i, n} \leq t<t_{i+1, n} \\ 0, & t=b\end{cases}
$$

$\left\{f_{n}\right\}_{n \in \mathbb{N}}$ converge to $f_{\alpha}^{\prime}$ a.e. on $[a, b]$. It is sufficient to prove $\left\{f_{n}\right\} \rightarrow f_{\alpha}^{\prime}$ in those points where $f$ is $\alpha$ differentiable and different from $t_{i, n}, i=0, \cdots, n$ for $n \in \mathbb{N}$, i.e., in

$$
\begin{aligned}
\mathcal{A} & =\left\{t \in[a, b] / f_{\alpha}^{\prime}(t) \text { exists }\right\} \\
& -\left\{t_{i, n} / n \in \mathbb{N}, i=0,1, \cdots, n\right\}
\end{aligned}
$$

For $t \in \mathcal{A}$, and each $n \in \mathbb{N}$, there exists $k \in\{0, \cdots, n\}$ such that $t_{k, n} \leq t<t_{k+1, n}$, so

$$
\begin{aligned}
f_{n}(t)= & \frac{f\left(t_{k+1, n}\right)-f\left(t_{k, n}\right)}{\alpha\left(t_{k+1, n}\right)-\alpha\left(t_{k, n}\right)} \\
= & \frac{\alpha\left(t_{k+1, n}\right)-\alpha(t)}{\alpha\left(t_{k+1, n}\right)-\alpha\left(t_{k, n}\right)} \frac{f\left(t_{k+1, n}\right)-f(t)}{\alpha\left(t_{k+1, n}\right)-\alpha(t)} \\
& +\frac{\alpha(t)-\alpha\left(t_{k, n}\right)}{\alpha\left(t_{k+1, n}\right)-\alpha\left(t_{k, n}\right)} \frac{f(t)-f\left(t_{k, n}\right)}{\alpha(t)-\alpha\left(t_{k, n}\right)} .
\end{aligned}
$$


Therefore, $f_{n}(t)$ is a convex combination of points

$$
\frac{f\left(t_{k+1, n}\right)-f(t)}{\alpha\left(t_{k+1, n}\right)-\alpha(t)} \text { and } \frac{f(t)-f\left(t_{k, n}\right)}{\alpha(t)-\alpha\left(t_{k, n}\right)} \text {. }
$$

Now if $n \rightarrow \infty$, then $t_{k, n} \rightarrow t$ and $t_{k+1, n} \rightarrow t$ and since $f$ is $\alpha$-differentiable for $t$, the expressions

$$
\frac{f\left(t_{k+1, n}\right)-f(t)}{\alpha\left(t_{k+1, n}\right)-\alpha(t)} \text { and } \frac{f(t)-f\left(t_{k, n}\right)}{\alpha(t)-\alpha\left(t_{k, n}\right)} \text {. }
$$

tend $f_{\alpha}^{\prime}(t)$ to which is $\alpha$-differentiable from $f$ in $t$. So results

$$
\lim _{n \rightarrow \infty} f_{n}(t)=f_{\alpha}^{\prime}(t)(t \in \mathcal{A} \text { a.e. in }[a, b]) .
$$

Since $\varphi$ is continuous, we have

$$
\lim _{n \rightarrow \infty} \varphi\left(\left|f_{n}(t)\right|\right)=\varphi\left(\lim _{n \rightarrow \infty}\left|f_{n}(t)\right|\right)=\varphi\left(\left|f_{\alpha}^{\prime}(t)\right|\right) \quad t \in \mathcal{A} .
$$

Using the Fatou's Lemma and definition of $f_{n}^{\prime}$ sequence, results that

$$
\begin{aligned}
\int_{a}^{b} \varphi\left(\left|f_{\alpha}^{\prime}(t)\right|\right) \mathrm{d} \alpha(t) & =\int_{a_{n \rightarrow \infty}^{b}}^{b} \lim _{n \rightarrow \infty} \varphi\left(\left|f_{n}(t)\right|\right) \mathrm{d} \alpha(t) \\
& \leq \liminf _{n \rightarrow \infty} \int_{a}^{b} \varphi\left(\left|f_{n}(t)\right|\right) \mathrm{d} \alpha(t) \\
& =\liminf _{n \rightarrow \infty} \sum_{i=0}^{n-1} \int_{t_{i, n}}^{t_{i+1, n}} \varphi\left(\left|f_{n}(t)\right|\right) \mathrm{d} \alpha(t) .
\end{aligned}
$$

By definition from $f_{n}$

$$
\begin{aligned}
& =\liminf _{n \rightarrow \infty} \sum_{i=0}^{n-1} \int_{t_{i, n}}^{t_{i+1, n}} \varphi\left(\frac{\left|f\left(t_{i+1, n}\right)-f\left(t_{i, n}\right)\right|}{\left|\alpha\left(t_{i+1, n}\right)-\alpha\left(t_{i, n}\right)\right|}\right)\left|\alpha\left(t_{i+1, n}\right)-\alpha\left(t_{i, n}\right)\right| \\
& =\liminf _{n \rightarrow \infty} \sum_{i=0}^{n-1} \varphi\left(\frac{\left|f\left(t_{i+1, n}\right)-f\left(t_{i, n}\right)\right|}{\left|\alpha\left(t_{i+1, n}\right)-\alpha\left(t_{i, n}\right)\right|}\right) \int_{t_{i, n}}^{t_{i+1, n}} \mathrm{~d} \alpha(t) \\
& =\liminf _{n \rightarrow \infty} \sum_{i=0}^{n-1} \varphi\left(\frac{\left|f\left(t_{i+1, n}\right)-f\left(t_{i, n}\right)\right|}{\left|\alpha\left(t_{i+1, n}\right)-\alpha\left(t_{i, n}\right)\right|}\left|\alpha\left(t_{i+1, n}\right)-\alpha\left(t_{i, n}\right)\right|\right. \\
& \leq V_{\alpha}(f,[a, b])<+\infty .
\end{aligned}
$$

which is what we wished to demonstrate.

Corollary 3.7 Let $\varphi \in \mathcal{N}$ such that satisfies the $\infty_{1}$ condition, then $f \in R V_{\varphi}(I)$ if and only if $f$ is $\alpha$-absolutely continuous on $[a, b]$ and

$$
\int_{a}^{b} \varphi\left(\left|f_{\alpha}^{\prime}(x)\right|\right) \mathrm{d} \alpha(x)<+\infty .
$$

Also

$$
\int_{a}^{b} \varphi\left(\left|f_{\alpha}^{\prime}(x)\right|\right) \mathrm{d} \alpha(x)=R V_{\varphi}(f,[a, b], \alpha) .
$$

Corollary 3.8 Let $\varphi \in \mathcal{N}$ such that satisfies the $\infty_{1}$ condition. If $f \in R V_{\varphi}(I)$, then $f$ is $\alpha$-absolutely continuous on $[a, b]$ and

$$
\int_{a}^{b} \varphi\left(\left|f_{\alpha}^{\prime}(x)\right|\right) \mathrm{d} \alpha(x)=R V_{\varphi}(f,[a, b], \alpha) .
$$

\section{Set-Valued Function}

Let $c c(X)$ be the family of all non-empty convex compact subsets of $X$ and $D$ be the Hausdorff metric in $c c(X)$, i.e.,

$$
D(A, B):=\inf \{t>0: A \subseteq B+t S, B \subseteq A+t S\},
$$

where $S=\{y \in X:\|y\| \leq 1\}$, or equivalently,

$$
D(A, B)=\max \{e(A, B), e(A, B): A, B \in c c(X)\},
$$

where

$$
\left\{\begin{array}{l}
e(A, B)=\sup \{d(x, B): x \in A\}, \\
d(x, B)=\inf \{d(x, y): y \in B\} .
\end{array}\right.
$$

Definition 4.1 Let $\varphi \in \mathcal{N}, \alpha$ a fixed continuous strictly increasing function and $F:[a, b] \rightarrow c c(X)$. We say that $F$ has bounded $\varphi$-variation in the sense of Riesz if

$$
\begin{aligned}
& W_{\varphi}(F,[a, b], \alpha) \\
& :=\sup _{\pi} \sum_{i=1}^{n} \varphi\left(\frac{D\left(F\left(t_{i}\right), F\left(t_{i-1}\right)\right)}{\left|\alpha\left(t_{i}\right)-\alpha\left(t_{i-1}\right)\right|}\right)\left|\alpha\left(t_{i}\right)-\alpha\left(t_{i-1}\right)\right|<\infty,
\end{aligned}
$$

where the supremum is taken over all partitions $\pi$ of $[a, b]$.

\section{Definition 4.2 Denote by}

$$
\begin{aligned}
& R W_{\varphi}^{*}(F,[a, b], \alpha) \\
& :=\left\{F:[a, b] \rightarrow c c(X): W_{\varphi}(F,[a, b], \alpha)<\infty\right\}
\end{aligned}
$$

and

$$
\begin{aligned}
& R W_{\varphi}(F,[a, b], \alpha):=\{F:[a, b] \\
& \left.\rightarrow c c(X): R W_{\varphi}^{*}(\lambda F)<\infty \text { for some } \lambda>0\right\},
\end{aligned}
$$

both equipped with the metric

$$
\begin{aligned}
& D_{\varphi}\left(F_{1}, F_{2}\right):=D\left(F_{1}(a), F_{2}(a)\right) \\
& +\inf \left\{\varepsilon>0: W_{\varphi}\left(F_{1} / \varepsilon, F_{2} / \varepsilon\right) \leq 1\right\},
\end{aligned}
$$

where

$$
\begin{aligned}
& W_{\varphi}\left(F_{1}, F_{2}\right) \\
& =\sup _{\pi} \sum_{i=1}^{n} \varphi\left(\frac{D\left(F_{1}\left(t_{i}\right)+F_{2}\left(t_{i-1}\right), F_{1}\left(t_{i-1}\right)+F_{2}\left(t_{i}\right)\right)}{\left|\alpha\left(t_{i}\right)-\alpha\left(t_{i-1}\right)\right|}\right) \\
& \cdot\left|\alpha\left(t_{i}\right)-\alpha\left(t_{i-1}\right)\right| .
\end{aligned}
$$

Now, let $(X,\|\cdot\|),(Y,\|\cdot\|)$ be two normed spaces and $K$ be a convex cone in $X$. Given a set-valued function $H:[a, b] \times K \rightarrow c c(Y)$ we consider the Nemytskii operator $N: K^{[a, b]} \rightarrow Y^{[a, b]}$ generated by $H$, that is 
the composition operator defined by:

$$
(N f)(t):=H(t, f(t)), f:[a, b] \rightarrow K ; t \in[a, b] .
$$

We denote by $L(K ; c c(Y))$ the space of all setvalued function $A: K \rightarrow c c(Y)$, i.e., additive and positively homogeneous, we say that $A$ is linear if $A \in L(K ; c c(Y))$.

In the proof of the main results of this paper, we will use some facts which we list here as lemmas.

Lemma 4.3 ([19]) Let $(X,\|\cdot\|)$ be a normed space and let $A, B, C$ be subsets of $X$. If $A, B$ are convex compact and $C$ is non-empty and bounded, then

$$
D(A+C, B+C)=D(A, B) \text {. }
$$

Lemma 4.4 ([20]) Let $(X,\|\cdot\|),(Y,\|\cdot\|)$ be normed spaces and $K$ be a convex cone in $X$. A set-valued function $F: K \rightarrow c c(Y)$ satisfies the Jensen equation

$$
F\left(\frac{x+y}{2}\right)=\frac{1}{2}(F(x)+F(y)), x, y \in K,
$$

if and only if there exists an additive set-valued function $A: K \rightarrow c c(Y)$ and a set $B \in c c(Y)$ such that

$$
F(x)=A(t)+B, \quad x \in K .
$$

We will extend the results of Aziz, Guerrero, Merentes and Sánchez given in [8] and [21] to set-valued functions of $\varphi$-bounded variation with respect to the weight function $\alpha$.

\section{Main Results}

Lemma 5.1 If $\varphi \in \mathcal{N}$ such that satisfies the $\infty_{1}$ condition and

$$
F \in R W_{\varphi}([a, b] ; c c(Y), \alpha),
$$

then $F:[a, b] \rightarrow c c(X)$ is continuous.

Proof. Since $F \in R W_{\varphi}([a, b], \alpha)$, exists $M>0$ such that

$$
\sum_{i=1}^{n} \varphi\left(\frac{D\left(F\left(t_{i}\right), F\left(t_{i-1}\right)\right)}{\left|\alpha\left(t_{i}\right)-\alpha\left(t_{i-1}\right)\right|}\right)\left|\alpha\left(t_{i}\right)-\alpha\left(t_{i-1}\right)\right| \leq M,
$$

for all partitions of $[a, b]$, in particular given $t, t_{0} \in[a, b]$, we have

$$
\varphi\left(\frac{D\left(F(t), F\left(t_{0}\right)\right)}{\left|\alpha(t)-\alpha\left(t_{0}\right)\right|}\right)\left|\alpha(t)-\alpha\left(t_{0}\right)\right| \leq M .
$$

Since $\varphi$ is convex $\varphi$-function, from the last inequa- lity, we get

$$
D\left(F(t), F\left(t_{0}\right)\right) \leq \frac{\varphi^{-1}\left(\frac{M}{\left|\alpha(t)-\alpha\left(t_{0}\right)\right|}\right)}{\frac{1}{\left|\alpha(t)-\alpha\left(t_{0}\right)\right|}} .
$$

By (1),

$$
\begin{aligned}
& \lim _{t \rightarrow t_{0}} D\left(F(t), F\left(t_{0}\right)\right) \\
& \leq \lim _{t \rightarrow t_{0}} \frac{\varphi^{-1}\left(\frac{M}{\left|\alpha(t)-\alpha\left(t_{0}\right)\right|}\right)}{\left|\alpha(t)-\alpha\left(t_{0}\right)\right|}=\lim _{\rho \rightarrow \infty} \frac{M \rho}{\varphi(\rho)}=0 .
\end{aligned}
$$

This proves the continuity of $F$ at $t_{0}$. Thus $F$ is continuous on $[a, b]$.

Now, we are ready to formulate the main result of this work.

Main Theorem 5.2 Let $(X,\|\cdot\|),(Y,\|\cdot\|)$ be normed spaces, $K$ be a convex cone in $X$ and $\varphi_{1}, \varphi_{2}$ be two convex $\varphi$-functions in $X$, strictly increasing, that satisfy $\infty_{1}$ condition and such that there exists constants $c$ and $T_{0}$ with $\varphi_{2}(t) \leq \varphi_{1}(c t)$ for all $t \geq T_{0}$. If the Nemitskii operator $N$ generated by a set-valued function $H:[a, b] \times K \rightarrow c c(Y)$ maps the space

$R V_{\varphi_{1}}(f,[a, b], \alpha ; K)$ into the space

$R W_{\varphi_{2}}(f,[a, b], \alpha ; c c(Y))$ and if it is globally Lipschitz, then the set-valued function $H$ satisfies the following conditions:

1) For every $t \in[a, b]$ there exists $M(t) \in[0,+\infty)$, such that

$$
D(H(t, x), H(t, y)) \leq M(t)\|x-y\|(x, y \in X) .
$$

2) There are functions $A:[a, b] \rightarrow L(K, c c(Y))$ and $B \in R W_{\varphi_{2}}(f,[a, b], \alpha ; c c(Y))$ such that

$$
H(t, x)=A(t) x+B(t) \quad(t \in[a, b], x \in K)
$$

Proof. 1) Since $N$ is globally Lipschitz, there exists a constant $M \in[0,+\infty)$ such that

$$
\begin{aligned}
& D_{\varphi_{2}}\left(N f_{1}, N f_{2}\right) \\
& \leq M\left\|f_{1}-f_{2}\right\|_{\varphi_{1}}\left(f_{1}, f_{2} \in R V_{\varphi_{1}}([a, b], \alpha ; K)\right) .
\end{aligned}
$$

Using the definitions of the operator $N$ and metric $D_{\varphi_{2}}$ we have

$$
\begin{aligned}
& D\left(N f_{1}(a), N f_{2}(a)\right)+\inf \left\{\varepsilon>0: \sup _{\pi} \sum_{i=1}^{n} \varphi_{2}\left(\frac{D\left(h_{t_{i}, t_{i-1}} N_{f_{1}, f_{2}}, h_{t_{i-1}, t_{i}} N_{f_{1}, f_{2}}\right)}{\varepsilon\left|\alpha\left(t_{i}\right)-\alpha\left(t_{i-1}\right)\right|}\right)\left|\alpha\left(t_{i}\right)-\alpha\left(t_{i-1}\right)\right| \leq 1\right\} \\
& \leq M\left\|f_{1}-f_{2}\right\|_{\varphi_{1}}\left(f_{1}, f_{2} \in R V_{\varphi_{1}}([a, b], \alpha ; K)\right),
\end{aligned}
$$


where $h_{s, t} N_{f_{1}, f_{2}}:=\left(N f_{1}\right)(s)+\left(N F_{2}\right)(t)$. In particular,

$$
\inf \left\{\varepsilon>0: \varphi_{2}\left(\frac{D\left(d_{f_{1}, f_{2}}(H, t, \bar{t}), d_{f_{1}, f_{2}}(H, \bar{t}, t)\right)}{\varepsilon|\alpha(\bar{t})-\alpha(t)|}\right)|\alpha(\bar{t})-\alpha(t)| \leq 1\right\} \leq M\left\|f_{1}-f_{2}\right\|_{\varphi_{1}},
$$

for all $f_{1}, f_{2} \in R V_{\varphi_{1}}([a, b], \alpha ; K)$ and $t, \bar{t} \in[a, b]$, $t \neq \bar{t}$, where

$$
d_{f_{1}, f_{2}}(H, s, t)=H\left(s, f_{1}(s)\right)+H\left(t, f_{2}(t)\right) .
$$

$$
\varphi_{i}\left(\varphi_{i}\left(\frac{1}{|\alpha(\bar{t})-\alpha(t)|}\right)\right)|\alpha(\bar{t})-\alpha(t)|=1, \quad i=1,2,
$$

Since $\varphi_{1}$ and $\varphi_{2}$ satisfy

we obtain

$$
\inf \left\{\varepsilon>0: \varphi_{2}\left(\frac{D\left(d_{f_{1}, f_{2}}(H, t, \bar{t}), d_{f_{1}, f_{2}}(H, \bar{t}, t)\right)}{\varepsilon|\alpha(\bar{t})-\alpha(t)|}\right)|\alpha(\bar{t})-\alpha(t)| \leq 1\right\}=D\left(d_{f_{1}, f_{2}}(H, t, \bar{t}), d_{f_{1}, f_{2}}(H, \bar{t}, t)\right)
$$

Therefore

$$
D\left(d_{f_{1}, f_{2}}(H, t, \bar{t}), d_{f_{1}, f_{2}}(H, \bar{t}, t)\right) \leq M\left\|f_{1}-f_{2}\right\|_{\varphi_{1}}|\alpha(\bar{t})-\alpha(t)| \varphi_{2}\left(\frac{1}{|\alpha(\bar{t})-\alpha(t)|}\right) .
$$

Define the auxiliary function $\eta:[a, b] \rightarrow[0,1]$ by:

$$
\eta(\tau):= \begin{cases}\frac{\alpha(\tau)-\alpha(a)}{\alpha(t)-\alpha(a)}, & a \leq \tau \leq t \\ 1, & t \leq \tau \leq b\end{cases}
$$

Then $\eta \in R V_{\varphi_{1}}([a, b], \alpha)$ and

$$
\begin{aligned}
& V_{\varphi_{1}}(\eta,[a, b], \alpha)=\varphi_{1}\left(\frac{1}{|\alpha(t)-\alpha(a)|}\right)|\alpha(t)-\alpha(a)| . \quad \text { Then the functions } f_{i} \in R V_{\varphi_{1}}([a, b], \alpha) \\
&\left\|f_{1}-f_{2}\right\|_{\varphi_{1}}=\left\|f_{1}(a)-f_{2}(a)\right\| \\
&+\inf \left\{\varepsilon>0: \sup _{\pi} \sum_{i=1}^{n} \varphi_{1}\left(\frac{\left\|\left(f_{1}-f_{2}\right)\left(t_{i}\right)-\left(f_{1}-f_{2}\right)\left(t_{i-1}\right)\right\|}{\varepsilon\left|\alpha\left(t_{i}\right)-\alpha\left(t_{i-1}\right)\right|}\right)\left|\alpha\left(t_{i}\right)-\alpha\left(t_{i-1}\right)\right| \leq 1\right\}
\end{aligned}
$$

Let us fix $x, y \in K$ and define the functions $f_{i}:[a, b] \rightarrow K \quad(i=1,2)$ by:

$$
\begin{aligned}
& f_{1}(\tau):=x, \\
& f_{2}(\tau):=\eta(\tau)(y-x)+x, \\
& \tau \in[a, b] .
\end{aligned}
$$

Then the functions $f_{i} \in R V_{\varphi_{1}}([a, b], \alpha ; K) \quad(i=1,2)$

From the definition of $f_{1}$ and $f_{2}$, we have

$$
\left\|f_{1}-f_{2}\right\|_{\varphi_{1}}=\inf \left\{\varepsilon>0: \varphi_{1}\left(\frac{\|x-y\|}{\varepsilon|\alpha(t)-\alpha(a)|}\right)|\alpha(t)-\alpha(a)| \leq 1\right\} .
$$

From (16), we get

$$
\inf \left\{\varepsilon>0: \varphi_{1}\left(\frac{\|x-y\|}{\varepsilon|\alpha(t)-\alpha(a)|}\right)|\alpha(t)-\alpha(a)|\right\}=\frac{\|x-y\|}{|\alpha(t)-\alpha(a)| \varphi_{1}^{-1}\left(\frac{1}{|\alpha(t)-\alpha(a)|}\right)}
$$

Hence, 


$$
D\left(d_{f_{1}, f_{2}}(H, t, \bar{t}), d_{f_{1}, f_{2}}(H, \bar{t}, t)\right) \leq \frac{M|\alpha(\bar{t})-\alpha(t)| \varphi_{2}^{-1}\left(\frac{1}{|\alpha(\bar{t})-\alpha(t)|}\right) \| x-y \mid}{|\alpha(t)-\alpha(a)| \varphi_{1}^{-1}\left(\frac{1}{|\alpha(t)-\alpha(a)|}\right)}
$$

Hence, substituting in inequality (5) the particular functions $f_{i} \quad(i=1,2)$ defined by (19) and taking $\alpha(\bar{t})=\alpha(a)$ in (23), we obtain

$$
D(H(t, x)+H(a, x), H(a, x)+H(t, y)) \leq M \frac{\varphi_{2}^{-1}\left(\frac{1}{|\alpha(t)-\alpha(a)|}\right)}{\varphi_{1}^{-1}\left(\frac{1}{|\alpha(t)-\alpha(a)|}\right)}\|x-y\|,
$$

for all $t \in[a, b], x, y \in K$.

By Lemma 4.3 and the inequality (24), we have

$$
D(H(t, x), H(t, y)) \leq M \frac{\varphi_{2}^{-1}\left(\frac{1}{|\alpha(t)-\alpha(a)|}\right)}{\varphi_{1}^{-1}\left(\frac{1}{|\alpha(t)-\alpha(a)|}\right)}\|x-y\|,
$$

for all $t \in[a, b], x, y \in K$.

$$
\eta_{1}(\tau):=\frac{\alpha(\tau)-\alpha(a)}{\alpha(b)-\alpha(a)}, \quad \tau \in[a, b] .
$$

Then the function $\eta_{1} \in R V_{\varphi_{1}}([a, b], \alpha)$ and

$$
V_{\varphi_{1}}\left(\eta_{1}\right)=\varphi_{1}\left(\frac{1}{|\alpha(b)-\alpha(a)|}\right)|\alpha(b)-\alpha(a)| .
$$

Let us fix $x, y \in K$ and define the functions $f_{i}:[a, b] \rightarrow K \quad(i=1,2)$ by

$$
f_{1}(\tau):=x, f_{2}(\tau):=\eta_{1}(\tau)(x-y)+y ; \tau \in[a, b] .
$$

Then the functions $f_{i} \in R V_{\varphi_{1}}([a, b], \alpha ; K)(i=1,2)$ and

Define the function $\eta_{1}:[a, b] \rightarrow[0,1]$ by

$$
\begin{aligned}
\left\|f_{1}-f_{2}\right\|_{\varphi_{1}} & =\|x-y\|+\inf \left\{\varepsilon>0: \varphi_{1}\left(\frac{\| x-y \mid}{\varepsilon|\alpha(b)-\alpha(a)|}\right)|\alpha(b)-\alpha(a)| \leq 1\right\} \\
& =\|x-y\|+\frac{\|x-y\|}{|\alpha(b)-\alpha(a)| \varphi_{1}^{-1}\left(\frac{1}{|\alpha(b)-\alpha(a)|}\right)} \\
& =\|x-y\|\left(1+\frac{1}{|\alpha(b)-\alpha(a)| \varphi_{1}^{-1}\left(\frac{1}{\mid \alpha(b)-\alpha(a)}\right)}\right)
\end{aligned}
$$

Substituting $\alpha(\bar{t})=\alpha(a)$ and $\alpha(t)=\alpha(b)$, and consider $\alpha=\alpha(b)-\alpha(a)$, we obtain

$$
\begin{aligned}
& D(H(b, x)+H(a, y), H(a, x)+H(b, x)) \\
& \leq M K\left(a, b, x, y, \varphi_{1}^{-1}, \varphi_{2}^{-1}\right)
\end{aligned}
$$

for all $x, y \in K$, where

$$
K\left(a, b, x, y, \varphi_{1}^{-1}, \varphi_{2}^{-1}\right)
$$

$$
=|\alpha| \varphi_{2}^{-1}\left(\frac{1}{|\alpha|}\right)\|x-y\|\left(1+\frac{1}{|\alpha| \varphi_{1}^{-1}\left(\frac{1}{|\alpha|}\right)}\right)
$$


By Lemma 4.3 and the above inequality, we get

$$
\begin{aligned}
& D(H(a, y), H(a, x)) \\
& \leq M|\alpha| \varphi_{2}^{-1}\left(\frac{1}{|\alpha|}\right)\|x-y\|\left(1+\frac{1}{|\alpha| \varphi_{1}^{-1}\left(\frac{1}{|\alpha|}\right)}\right)
\end{aligned}
$$

for all $x, y \in K$. Define the function $M:[a, b] \rightarrow \mathbb{R}$ by

$$
M(t)=\left\{\begin{array}{l}
M \frac{\varphi_{2}^{-1}\left(\frac{1}{|\alpha(t)-\alpha(a)|}\right)}{\varphi_{1}^{-1}\left(\frac{1}{|\alpha(t)-\alpha(a)|}\right)}, a<t \leq b \\
M|\alpha| \varphi_{2}^{-1}\left(\frac{1}{|\alpha|}\right)|| x-y||\left(1+\frac{1}{|\alpha| \varphi_{1}^{-1}\left(\frac{1}{|\alpha|}\right)}\right), t=a .
\end{array}\right.
$$

Hence

$$
\begin{aligned}
& D(H(t, x), H(t, y)) \\
& \leq M(t)\|x-y\|(x, y \in X, t \in[a, b]),
\end{aligned}
$$

and, consequently, for every $t \in[a, b]$ the function $H:[a, b] \times K \rightarrow c c(Y)$ is continuous.

This completes the proof of part 1).

Now we shall prove that $H$ satisfies equality 2).

Let us fix $t, t_{0} \in[a, b]$ such that $t_{0}<t$. Since the Nemytskii operator $N$ is globally Lipschitzian, there exists a constant $M$, such that

$$
\begin{aligned}
& D\left(d_{u, v}\left(H, t, t_{0}\right), d_{u, v}\left(H, t_{0}, t\right)\right) \\
\leq & M\|u-v\|_{\varphi_{1}}\left|\alpha\left(t_{0}\right)-\alpha(t)\right| \varphi_{2}\left(\frac{1}{\left|\alpha\left(t_{0}\right)-\alpha(t)\right|}\right),
\end{aligned}
$$

where $d_{u, v}(H, s, t)=H(s, u(s))+H(t, v(t))$. Define the function $\eta_{2}:[a, b] \rightarrow[0,1]$ by

$$
\eta_{2}(\tau)= \begin{cases}\frac{\alpha(\tau)-\alpha(a)}{\alpha\left(t_{0}\right)-\alpha(a)}, & a \leq \tau \leq t_{0}, \\ \frac{\alpha(t)-\alpha(\tau)}{\alpha(t)-\alpha\left(t_{0}\right)}, & t_{0} \leq \tau \leq t, \\ 0, & t \leq \tau \leq b .\end{cases}
$$

The function $\eta_{2} \in R V_{\varphi_{1}}([a, b], \alpha)$.

Let us fix $x, y \in K$ and define the functions $f_{i}:[a, b] \rightarrow K$ by

$$
\begin{cases}f_{1}(\tau):=\frac{1}{2} \eta_{2}(\tau) x+\left(1-\frac{1}{2} \eta_{2}(\tau)\right) y, & \tau \in[a, b] ; \\ f_{2}(\tau):=\frac{1}{2}\left(1+\eta_{2}(\tau)\right) x+\frac{1}{2}\left(1-\eta_{2}(\tau)\right) y, & \tau \in[a, b] .\end{cases}
$$

The functions $f_{i} \in R V_{\varphi_{1}}([a, b], \alpha ; K) \quad(i=1,2)$ and

$$
\left\|f_{1}-f_{2}\right\|_{\varphi_{1}}=\frac{\|x-y\|}{2} \text {. }
$$

Hence, substituting in the inequality (28) the particular functions $f_{i} \quad(i=1,2)$ defined by (29), we obtain

$$
\begin{aligned}
& D\left(H\left(t_{0}, x\right)+H(t, y), H\left(t_{0}, \frac{x+y}{2}\right)+H\left(t, \frac{x+y}{2}\right)\right) \\
& \leq \frac{1}{2} M\left|\alpha(t)-\alpha\left(t_{0}\right)\right| \varphi_{2}^{-1}\left(\frac{1}{\left|\alpha(t)-\alpha\left(t_{0}\right)\right|}\right)\|x-y\| .
\end{aligned}
$$

Since $N$ maps

$$
R V_{\varphi_{1}}([a, b], \alpha ; K) \text { into } R W_{\varphi_{2}}([a, b], \alpha ; c c(Y)),
$$
then $H(\cdot, z)$ is continuous for all $z \in K$. Hence letting $t_{0} \uparrow t$ in the inequality (30), we get

$$
\begin{aligned}
& D\left(H(t, x)+H(t, y), H\left(t, \frac{x+y}{2}\right)+H\left(t, \frac{x+y}{2}\right)\right) \\
& =0,
\end{aligned}
$$

for all $t \in[a, b]$ and $x, y \in K$.

Thus for all $t \in[a, b], x, y \in K$, we have

$$
H\left(t, \frac{x+y}{2}\right)+H\left(t, \frac{x+y}{2}\right)=H(t, x)+H(t, y) .
$$

Since $H$ is convex, we have

$$
H\left(t, \frac{x+y}{2}\right)=\frac{1}{2}[H(t, x)+H(t, y)],
$$

for all $t \in[a, b], x, y \in K$. Thus for all $t \in[a, b]$, the set-valued function $H(t, \cdot): K \rightarrow c c(Y)$ satisfies the Jensen Equation (33). Now by Lemma 4.4, there exists an additive set-valued function $A(t): K \rightarrow c c(Y)$ and a set $B(t) \in c c(Y)$, such that

$$
H(t, x)=A(t) x+B(t), \quad(x \in K, t \in[a, b]) .
$$

Substituting $H(t, x)=A(t) x+B(t)$ into inequality (13), we deduce that for all $t \in[a, b]$ there exists $M(t) \in[0,+\infty)$, such that

$$
D(A(t) x, A(t) y) \leq M(t)\|x-y\| \quad(x, y \in K),
$$

consequently, for every $t \in[a, b]$ the set-valued function $A(t): K \rightarrow c c(Y)$ is continuous, and

$$
A(t)(\cdot) \in L(K, c c(Y)) .
$$

Since $A(t)(\cdot)$ is additive and $0 \in K$, then $A(t)=\{0\}$ for all $t \in[a, b]$, thus $H(\cdot, 0)=B(\cdot)$. 
The Nemytskii operator $N$ maps the space $R V_{\varphi_{1}}([a, b], \alpha ; K)$ into the space $R W_{\varphi_{2}}([a, b] ; c c(Y))$, then

$$
H(\cdot, 0)=B(\cdot) \in R W_{\varphi_{2}}([a, b], \alpha ; K) .
$$

Consequently the set-valued function $H$ has to be of the form

$$
H(t, x)=A(t) x+B(t), \quad t \in[a, b], x \in K,
$$

where $A(t) \in L(K, c c(Y))$ and

$$
B \in R W_{\varphi_{2}}([a, b], \alpha ; c c(Y)) .
$$

Theorem 5.3 Let $(X,\|\cdot\|),(Y,\|\cdot\|)$ be normed spaces, $K$ a convex cone in $X$ and $\varphi_{1}, \varphi_{2}$ be two convex $\varphi$-functions in $X$, strictly increassing, satisfying $\infty_{1}$ condition and $\lim _{t \rightarrow \infty} \varphi_{2}^{-1}\left(\varphi_{1}(t)\right) / t=\infty$. If the Nemytskii operator $N$ generated by a set-valued function $H:[a, b] \times K \rightarrow c c(Y)$ maps the space $R V_{\varphi_{2}}([a, b] \alpha ; K)$ into the space $R W_{\varphi_{1}}([a, b], \alpha ; c c(Y))$ and if it is globally Lipschizian, then the set-valued function $H$ satisfies the following condition

$$
H(t, x)=H(t, 0)(t \in[a, b], x \in K) ;
$$

i.e., the Nemytskii operator is constant.

Proof. Since the Nemytskii operator $N$ is globally Lipschizian between $R V_{\varphi_{1}}([a, b], \alpha ; K)$ and the space $R W_{\varphi_{1}}([a, b], \alpha ; c c(Y))$, then there exists a constant $M$, such that

$$
\begin{aligned}
& D_{\varphi_{1}}\left(N f_{1}, N f_{2}\right) \\
& \leq M\left\|f_{1}-f\right\|_{2 \varphi_{2}}\left(f_{1}, f_{2} \in R V_{\varphi_{2}}([a, b], \alpha ; K)\right) .
\end{aligned}
$$

Let us fix $t, t_{0} \in[a, b]$ such that $t_{0}<t$. Using the definitions of the operator $N$ and of the metric $D_{\varphi_{1}}$, we have

$$
\begin{aligned}
& D\left(H\left(t, f_{1}(t)\right)+H\left(t_{0}, f_{2}\left(t_{0}\right)\right),\right. \\
& \left.H\left(t_{0}, f_{1}\left(t_{0}\right)\right)+H\left(t, f_{2}(t)\right)\right) \\
& \leq M\left|\alpha(t)-\alpha\left(t_{0}\right)\right|\left\|f_{1}-f_{2}\right\|_{\varphi_{2}} \varphi_{1}^{-1}\left(\frac{1}{\left|\alpha(t)-\alpha\left(t_{0}\right)\right|}\right) \\
& \left(f_{1}, f_{2} \in R V_{\varphi_{2}}([a, b], \alpha ; K)\right)
\end{aligned}
$$

Define the auxiliary function $\eta_{3}:[a, b] \rightarrow[0,1]$. by

$$
\eta_{3}(\tau):= \begin{cases}1, & a \leq \tau \leq t_{0}, \\ -\frac{\alpha(\tau)-\alpha(t)}{\alpha(t)-\alpha\left(t_{0}\right)}, & t_{0} \leq \tau \leq t, \\ 0, & t \leq \tau \leq b .\end{cases}
$$

The function $\eta_{3} \in R V_{\varphi_{2}}([a, b], \alpha)$ and

$$
V_{\varphi_{2}}\left(\eta_{3} ;[a, b]\right)=\left|\alpha(t)-\alpha\left(t_{0}\right)\right| \varphi_{2}^{-1}\left(\frac{1}{\left|\alpha(t)-\alpha\left(t_{0}\right)\right|}\right) \text {. }
$$

Let us fix $x \in K$ and define the functions $f_{i}:[a, b] \rightarrow K \quad(i=1,2)$ by

$$
f_{1}(\tau):=x, \quad f_{2}(\tau):=\eta_{3}(\tau) x, \quad \tau \in[a, b] .
$$

The functions $f_{i} \in R V_{\varphi_{2}}([a, b], \alpha ; K) \quad(i=1,2)$ and

$$
\begin{aligned}
\left\|f_{1}-f_{2}\right\|_{\varphi_{2}} & =\left\|f_{1}(a)-f_{2}(a)\right\|+\inf \left\{\varepsilon>0: \sup _{\pi} \sum_{i=1}^{n} \varphi_{2}\left(\frac{\left\|\left(f_{1}-f_{2}\right)\left(t_{i}\right)-\left(f_{1}-f_{2}\right)\left(t_{i-1}\right)\right\|}{\varepsilon\left|\alpha\left(t_{i}\right)-\alpha\left(t_{i-1}\right)\right|}\right)\left|\alpha\left(t_{i}\right)-\alpha\left(t_{i-1}\right)\right| \leq 1\right\} \\
& =\inf \left\{\varepsilon>0: \varphi_{2}\left(\frac{\|x\|}{\varepsilon\left|\alpha(t)-\alpha\left(t_{0}\right)\right|}\right)\left|\alpha(t)-\alpha\left(t_{0}\right)\right| \leq 1\right\} \\
& =\frac{\|x\|}{\left|\alpha(t)-\alpha\left(t_{0}\right)\right| \varphi_{2}^{-1}\left(\frac{1}{\left|\alpha(t)-\alpha\left(t_{0}\right)\right|}\right)} .
\end{aligned}
$$

Hence, substituting in the inequality (36) the auxiliary functions $f_{i} \quad(i=1,2)$ defined by (37), we obtain

$$
D\left(H(t, x)+H\left(t_{0}, x\right), H\left(t_{0}, x\right)+H(t, 0)\right) \leq M\left|\alpha(t)-\alpha\left(t_{0}\right)\right| \frac{\varphi_{1}^{-1}\left(\frac{1}{\left|\alpha(t)-\alpha\left(t_{0}\right)\right|}\right)}{\left|\alpha(t)-\alpha\left(t_{0}\right)\right| \varphi_{2}^{-1}\left(\frac{1}{\left|\alpha(t)-\alpha\left(t_{0}\right)\right|}\right)}\|x\| .
$$

By Lemma 4.3 and the above inequality, we get 


$$
D(H(t, x), H(t, 0)) \leq M\left|\alpha(t)-\alpha\left(t_{0}\right)\right| \frac{\varphi_{1}^{-1}\left(\frac{1}{\left|\alpha(t)-\alpha\left(t_{0}\right)\right|}\right)}{\left|\alpha(t)-\alpha\left(t_{0}\right)\right| \varphi_{2}^{-1}\left(\frac{1}{\left|\alpha(t)-\alpha\left(t_{0}\right)\right|}\right)}\|x\| .
$$

Since $\lim _{t \rightarrow \infty} \varphi_{2}^{-1}\left(\varphi_{1}(t)\right) / t=\infty$, letting $t_{0} \uparrow t$ in the above inequality, we have

$$
D(H(t, x), H(t, 0))=0 .
$$

Thus for all $t \in[a, b]$ and for all $x \in K$, we get

$$
H(t, x)=H(t, 0) .
$$

Theorem 5.4 Let $(X,\|\cdot\|),(Y,\|\cdot\|)$ be normed spaces, $K$ a convex cone in $X$ and $\varphi$ be a convex $\varphi$ function in $X$ satisfying the $\infty_{1}$ condition. If the Nemytskii operator $N$ generated by a set-valued function $H:[a, b] \times K \rightarrow c c(Y)$ maps the space $R V_{\varphi}([a, b], \alpha ; K)$ into the space $B W([a, b] ; c c(Y))$ and if it is globally Lipschizian, then the left regularization $H^{*}:[a, b] \times K \rightarrow c c(Y)$ of the function $H$ defined by

$$
H^{*}(t, x):= \begin{cases}H(t, x), & t \in(a, b], x \in K ; \\ \lim _{s \downarrow a} H(s, x), & t=a, x \in K,\end{cases}
$$

satisfies the following conditions:

- for all $t \in[a, b]$ there exists $M(t)$, such that
$D\left(H^{*}(t, x), H^{*}(t, y)\right) \leq M(t)\|x-y\| \quad(x, y \in K)$.

- $H^{*}(t, x)=A(t) x+B(t) \quad(t \in[a, b] x \in K)$, where $A(t)$ is a linear continuous set-valued function, and $B \in B W([a, b] ; c c(Y))$.

Proof. We take $t \in[a, b]$, and define the auxiliary function $\eta_{4}:[a, b] \rightarrow[0,1]$ by:

$$
\eta_{4}(\tau):= \begin{cases}1, & a \leq \tau \leq t, \\ \frac{\alpha(\tau)-\alpha(b)}{\alpha(t)-\alpha(b)}, & t \leq \tau \leq b .\end{cases}
$$

The function $\eta_{4} \in R V_{\varphi}([a, b], \alpha ; K)$ and

$$
V_{\varphi}\left(\eta_{4},[a, b]\right)=\varphi\left(\frac{1}{|\alpha(b)-\alpha(t)|}\right)|\alpha(b)-\alpha(t)| .
$$

Let us fix $x, y \in K$ and define the functions $f_{i}:[a, b] \rightarrow K \quad(i=1,2)$ by

$$
f_{1}(\tau):=x, f_{2}(\tau):=\eta_{4}(\tau)(y-x)+x, \quad \tau \in[a, b] .
$$

The functions $f_{i} \in R V_{\varphi}([a, b], \alpha ; K) \quad(i=1,2)$ and

$$
\left\|f_{1}-f_{2}\right\|_{\varphi}=\left\|f_{1}(a)-f_{2}(a)\right\|+\inf \left\{\varepsilon>0: \sup _{\pi} \sum_{i=1}^{n} \varphi\left(\frac{\left\|\left(f_{1}-f_{2}\right)\left(t_{i}\right)-\left(f_{1}-f_{2}\right)\left(t_{i-1}\right)\right\|}{\varepsilon\left|\alpha\left(t_{i}\right)-\alpha\left(t_{i-1}\right)\right|}\right)\left|\alpha\left(t_{i}\right)-\alpha\left(t_{i-1}\right)\right| \leq 1\right\}
$$

From the definition of $f_{1}$ and $f_{2}$, we obtain

$$
\left\|f_{1}-f_{2}\right\|_{\varphi}=\|x-y\|\left(1+\frac{1}{|\alpha(b)-\alpha(t)| \varphi^{-1}\left(\frac{1}{\mid \alpha(b)-\alpha(t)}\right)}\right) \text {. }
$$

Since the Nemytskii operator $N$ is globally Lipschitzian between
$R V_{\varphi}([a, b], \alpha ; K)$ and $B W([a, b] ; c c(Y))$, then there exists a constant $M$, such that

$$
D\left(H\left(b, f_{1}(b)\right)+H\left(t, f_{2}(t)\right), H\left(t, f_{1}(t)\right)+H\left(b, f_{2}(b)\right)\right) \leq M\left\|f_{1}-f_{2}\right\|_{\varphi} .
$$

for $f_{1}, f_{2} \in R V_{\varphi}([a, b], \alpha ; K)$. By Lemma 4.3, substituting the particular functions $f_{i}(i=1,2)$ defined by (38) in the above inequality, we obtain

$$
D\left(H\left(b, f_{1}(b)\right)+H\left(t, f_{2}(t)\right), H\left(t, f_{1}(t)\right)+H\left(b, f_{2}(b)\right)\right) \leq M(t)\|x-y\|_{\varphi},
$$


for all $x, y \in K, t \in[a, b]$. By Lemma 4.3, we get

$$
D(H(t, x), H(t, y)) \leq M(t)\|x-y\|_{\varphi}
$$

for all $t \in[a, b)$ and $x, y \in K$.

In the case where $t=b$, by a similar reasoning as above, we obtain that there exists a constant $M(b)$, such that

$$
D(H(b, x), H(b, y)) \leq M(b)\|x-y\|_{\varphi}(x, y \in K) .
$$

Define the function $M:[a, b] \rightarrow \mathbb{R}$ by

$$
M(t)=\left\{\begin{array}{l}
M\left(1+\frac{1}{|\alpha(b)-\alpha(t)| \varphi^{-1}\left(\frac{1}{|\alpha(b)-\alpha(t)|}\right)}\right), \\
M(b), \quad a \leq t<b, \\
t=b .
\end{array}\right.
$$

Hence,

$$
\begin{aligned}
& D(H(t, x), H(t, y)) \leq M(t)\|x-y\|_{\varphi}, \\
& t \in[a, b], x, y \in K .
\end{aligned}
$$

By passing to the limit in the inequality (41) by the inequality (43) and the definition of $H^{*}$ we have for all $t \in[a, b]$ that there exists $M(t)$, such that

$$
\begin{aligned}
& D\left(H^{*}(t, x), H^{*}(t, y)\right) \leq M(t)\|x-y\|_{\varphi} \\
& (t \in[a, b] x, y \in K)
\end{aligned}
$$

Now we shall prove that $H^{*}$ satisfies the following equality

$$
H^{*}(t, x)=A(t) x+B(t) \quad(t \in[a, b], x \in K),
$$

where $A(t)$ is a linear continuous set-valued functions, and

$$
B \in B W([a, b] ; c c(Y)) .
$$

Let us fix $t, t_{0} \in[a, b], n \in \mathbb{N}$ such that $t_{0}<t$. De- fine the partition $\pi_{n}$ of the interval $\left[t_{0}, t\right]$ by

$$
\begin{aligned}
& \pi_{n}: a<t_{0}<t_{1}<\cdots<t_{2 n-1}<t_{2 n}=t, \\
& \text { where } t_{i}-t_{i-1}=\frac{t-t_{0}}{2 n}, \quad i=1,2, \cdots, 2 n .
\end{aligned}
$$

The Nemytskii operator $N$ is globally Lipschitzian between $R V_{\varphi}([a, b], \alpha ; K)$ and $B W([a, b] ; c c(Y))$, then there exists a constant $M>0$, such that

$$
\begin{aligned}
& \sum_{i=1}^{n} D\left(d_{f_{1}, f_{2}}\left(H, t_{2 i}, t_{2 i-1}\right), d_{f_{1}, f_{2}}\left(H, t_{2 i-1}, t_{2 i}\right)\right) \\
& \leq M\left\|f_{1}-f_{2}\right\|_{\varphi},
\end{aligned}
$$

where

$$
f_{1}, f_{2} \in R V_{\varphi}([a, b], \alpha ; K)
$$

and

$$
d_{f_{1}, f_{2}}(H, s, t)=H\left(s, f_{1}(s)\right)+H\left(t, f_{2}(t)\right) .
$$

We define the function $\eta_{5}:[a, b] \rightarrow[0,1]$ in the following way:

$$
\eta_{5}(\tau):= \begin{cases}0, & a \leq \tau \leq t_{0} \\ \frac{\alpha(\tau)-\alpha\left(t_{i-1}\right)}{\alpha\left(t_{i}\right)-\alpha\left(t_{i-1}\right)}, & t_{i-1} \leq \tau \leq t_{i}, i=1,3, \cdots, 2 n-1 ; \\ -\frac{\alpha(\tau)-\alpha\left(t_{i}\right)}{\alpha\left(t_{i}\right)-\alpha\left(t_{i-1}\right)}, & t_{i-1} \leq \tau \leq t_{i}, i=2,4, \cdots, 2 n \\ 0, & t \leq \tau \leq b .\end{cases}
$$

The function $\eta_{5} \in R V_{\varphi}([a, b], \alpha ; K)$ and

$$
V_{\varphi}\left(\eta_{5}, \alpha ;[a, b]\right)=\left|\alpha(t)-\alpha\left(t_{0}\right)\right| \varphi\left(\frac{2 n}{\mid \alpha(t)-\alpha\left(\left(t_{0}\right) \mid\right.}\right) .
$$

Let us fix $x, y \in K$ and define the functions $f_{i}:[a, b] \rightarrow K$ by: 


$$
\begin{cases}f_{1}(\tau):=\frac{1}{2} \eta_{5}(\tau) x+\left[1-\frac{1}{2} \eta_{5}(\tau)\right] y, & \tau \in[a, b] \\ f_{2}(\tau):=\frac{1}{2}\left[1+\eta_{5}(\tau)\right] x+\frac{1}{2}\left[1-\eta_{5}(\tau)\right] y, & \tau \in[a, b] .\end{cases}
$$

The functions $f_{i} \in R V_{\varphi}([a, b], \alpha ; K)(i=1,2)$ and

$$
\left\|f_{1}-f_{2}\right\|_{\varphi}=\frac{\|x-y\|}{2} .
$$

Substituting in the inequality (45) the particular functions $f_{i} \quad(i=1,2)$ defined in (46), we obtain

$$
\sum_{i=1}^{n} D\left(H\left(t_{2 i-1}, x\right)+H\left(t_{2 i}, y\right), H\left(t_{2 i-1}, \frac{x+y}{2}\right)+H\left(t_{2 i}, \frac{x+y}{2}\right)\right) \leq \frac{1}{2} M\|x-y\|_{\varphi} \quad x, y \in K .
$$

Since the Nemytskii operator $N$ maps the spaces $R V_{\varphi}([a, b], \alpha ; K)$ into $B W([a, b] ; c c(Y))$, then for all $z \in K$, the function $H(\cdot, z) \in B W([a, b] ; c c(Y))$. Letting $t_{0} \uparrow t$ in the inequality (47), we get

$$
\begin{aligned}
& D\left(H^{*}(t, x)+H^{*}(t, y), H^{*}\left(t, \frac{x+y}{2}\right)+H^{*}\left(t, \frac{x+y}{2}\right)\right) \\
\leq & \frac{M}{2 n}\|x-y\|_{\varphi} .
\end{aligned}
$$

for all $x, y \in K$ and $n \in \mathbb{N}$. By passing to the limit when $n \rightarrow \infty$, we get

$$
\begin{aligned}
& H^{*}\left(t, \frac{x+y}{2}\right)+H^{*}\left(t, \frac{x+y}{2}\right)=H^{*}(t, x)+H^{*}(t, y), \\
& t \in[a, b], x, y \in K .
\end{aligned}
$$

Since $H^{*}(t, x)$ is a convex function, then

$$
\begin{aligned}
& H^{*}\left(t, \frac{x+y}{2}\right)=\frac{1}{2}\left[H^{*}(t, x)+H^{*}(t, y)\right] \\
& (t \in[a, b], x, y \in K) .
\end{aligned}
$$

Thus for every $t \in[a, b]$, the set-valued function $H^{*}(t, \cdot): K \rightarrow c c(Y)$ satisfies the Jensen equation. By Lemma 4.4 and by the property (a) previously established, we get that for all $t \in[a, b]$ there exist an additive set-valued function $A(\cdot): K \rightarrow c c(Y)$ and a set $B(t) \in c c(Y)$, such that

$$
H^{*}(t, x)=A(t) x+B(t) \quad(t \in[a, b], x \in K) .
$$

By the same reasoning as in the proof of Theorem 5.2, we obtain that

$$
A(t)(\cdot) \in L(K, c c(Y)) \text { and } B \in B W([a, b] ; c c(Y)) .
$$

\section{Acknowledgements}

This research was partly supported by CDCHTA of Universidad de Los Andes under the project NURR-C-
547-12-05-B.

\section{REFERENCES}

[1] A. Smajdor and W. Smajdor, "Jensen Equation and Nemytskii Operator for Set-Valued Functions," Radovi Matematicki, Vol. 5, 1989, pp. 311-319.

[2] J. Matkwoski, "Functional Equation and Nemytskii Operators," Funkcialaj Ekvacioj, Vol. 25, No. 2, 1982, pp. 127-132.

[3] G. Zawadzka, "On Lipschitzian Operators of Substitution in the Space of Set-Valued Functions of Bounded Variation," Radovi Matematicki, Vol. 6, 1990, pp. 179-193.

[4] J. Matkwoski and J. Mis, "On a Characterization of Lipschitzian Operators of Substitution in the Space BV(a,b)," Mathematische Nachrichten. Vol. 117, No. 1, 1984, pp. 155-159. doi:10.1002/mana.3211170111

[5] N. Merentes and K. Nikodem, "On Nemytskii Operator and Set-Valued Functions of Bounded P-Variation," Radovi Matematicki, Vol. 8, 1992, pp. 139-145.

[6] N. Merentes and S. Rivas, "On Nemytskii Operator in the Space of Set-Valued Functions of Bounded P-Variation in the Sense of Riesz," Publicationes Mathematicae Debrecen, Vol. 47, No. 1-2, 1995, pp. 15-27.

[7] N. Merentes and J. L. Sánchez, "Characterization of Globally Lipschitz Nemytskii Operator between Spaces of Set-Valued Functions of Bounded $\varphi$-Variation in Sense of Riesz," Bulletin of the Polish Academy of Sciences Mathematics, Vol. 52, No. 4, 2004, pp. 417-430. doi: $10.4064 / \mathrm{ba} 52-4-8$

[8] W. Aziz, J. A. Guerrero, N. Merentes and J. L. Sánchez, "Nemytskii Operator in the Space of Set-Valued Functions of Bounded P-Variation," JMCSA, Vol. 4, No. 1, 2011, pp. 85-94.

[9] V. V. Chistyakov, "Lipschitzian Superposition Operators between Spaces of Functions of Bounded Generalized Variation with Weight," Journal of Applied Analysis, Vol. 6, No. 2, 2000, pp. 173-186. doi:10.1515/JAA.2000.173

[10] F. Riesz, "Untersuchugen Über Systeme Integrierbarer Funktionen," Mathematische Annalen, Vol. 69, No. 4, 1910, pp. 449-497. doi:10.1007/BF01457637 
[11] Yu. T. Medvedev, "A Generalization of a Theorem of F. Riesz," Uspekhi Matematicheskikh Nauk, Vol. 6, No. 8, 1953, pp. 115-118.

[12] Z. Cibertowicz and W. Matuszewska, "Functions of Bounded Generalized Variations," Commentationes Mathematicae (Prace Matematyczne), Vol. 20, No. 1, 1977, pp. 29-52.

[13] W. A. J. Luxemburg, "Banach Function Spaces," Ph.D. Dissertation, Technische Hogeschool te Delft, 1955.

[14] H. Nakano, "Modulared Semi-Ordered Spaces," Tokyo, 1950.

[15] L. Maligranda and W. Orlicz, "On Some Properties of Functions of Generalized Variation," Monatshefte für Mathematik, Vol. 104, No. 1, 1987, pp. 53-65.

[16] F. Riesz and B. Sz. Nagy, "Functional Analysis," Ungar, New York, 1955.

[17] M. C. Chakrabarty, "Some Results on AC- $\omega$ Functions,"
Fundamenta Mathematicae, Vol. 64, No. 2, 1969, pp 219-230.

[18] R. L. Jeffery, "Generalized Integrals with Respect to Bounded Variation," Canadian Journal of Mathematics, Vol. 10, 1958, pp. 617-628.

[19] H. Rådström, "An Embedding Theorem for Space of Convex Sets," Proceedings of the American Mathematical Society, Vol. 3, No. 1, 1952, pp. 165-169.

[20] K. Nikodem, "K-Convex and K-Concave Set-Valued Functions," Zeszyty Naukowe Politechniki Łódzkiej, Vol. 559, 1989, pp. 210-225.

[21] W. Aziz, J. A. Guerrero and N. Merentes, "On Nemytskii Operator in the Space of Set-Valued Functions of Bounded $p$-Variation in the Sense of Riesz with Respect to the Weight Function," Fasciculi Mathematici, Vol. 50, 2013, in press. 